TRANSACTIONS OF THE

AMERICAN MATHEMATICAL SOCIETY

Volume 362, Number 6, June 2010, Pages 2811-2831

S 0002-9947(10)05006-3

Article electronically published on January 7, 2010

\title{
ORBIFOLDS AS DIFFEOLOGIES
}

\author{
PATRICK IGLESIAS, YAEL KARSHON, AND MOSHE ZADKA
}

\begin{abstract}
We consider orbifolds as diffeological spaces. This gives rise to a natural notion of differentiable maps between orbifolds, making them into a subcategory of diffeology. We prove that the diffeological approach to orbifolds is equivalent to Satake's notion of a V-manifold and to Haefliger's notion of an orbifold. This follows from a lemma: a diffeomorphism (in the diffeological sense) of finite linear quotients lifts to an equivariant diffeomorphism.
\end{abstract}

\section{INTRODUCTION}

An orbifold is a space that is locally modeled on quotients of $\mathbb{R}^{n}$ by finite linear group actions. The precise definition of its global structure is more complicated. The situation in the literature is somewhat problematic: different authors give definitions that are a priori different from each other, and the relations between them are not made clear.

We propose to approach orbifolds through the notion of a diffeology. A diffeology on a set $X$ specifies, for each whole number $m$ and each open subset $U$ of $\mathbb{R}^{m}$, which functions from $U$ to $X$ are differentiable. A diffeological space is a set equipped with a diffeology. A quotient of a diffeological space is again a diffeological space; a map to the quotient is declared to be differentiable if and only if it locally lifts. We define a diffeological orbifold to be a diffeological space that is locally diffeomorphic at each point to the quotient of $\mathbb{R}^{n}$ by a finite linear group action. For details, see Section 2 .

In Section 3 we show that a diffeomorphism (in the diffeological sense) between finite linear quotients of $\mathbb{R}^{n}$ lifts to an equivariant diffeomorphism. See Lemma 23. This fails for arbitrary differentiable maps; see Examples 24 and 25.

To illustrate that the diffeology retains rich information, in Section 4 we show that the stabilizers of a finite group action are a diffeological invariant of the quotient.

Orbifolds were originally introduced by Satake under the name "V-manifolds". Satake's definition is subtle and is often quoted imprecisely. We recall this definition in Section 5. A problem with the definition is that it does not lead to a satisfactory notion of $C^{\infty}$-maps. (In S2, page 469], Satake writes this footnote: "The notion of $C^{\infty}$-map thus defined is inconvenient in the point that a composite of two $C^{\infty}$. maps defined in a different choice of defining families is not always a $C^{\infty}$-map.")

Received by the editors August 20, 2007.

2010 Mathematics Subject Classification. Primary 57R18.

This research was partially supported by the Israel Science Foundation founded by the Academy of Sciences and Humanities, by the National Center for Scientific Research (CNRS, France), and by the National Science and Engineering Research Council of Canada (NSERC).

(C)2010 American Mathematical Society Reverts to public domain 28 years from publication 
A related problem with Satake's definition is that it is not a priori clear whether a V-manifold structure is local, that is, whether a V-manifold structure can be determined by its restrictions to the elements of an open covering. In diffeology, the notion of a differentiable map is completely natural, and, with it, diffeological orbifolds become a category. Also, the axioms of diffeology immediately imply that a diffeological orbifold structure is local.

In order to not increase the confusion in the literature, we verify that, under mild restrictions (Satake insisted that the space be Hausdorff and the finite groups act without reflections), our definition of a diffeological orbifold is equivalent to Satake's notion of a V-manifold. See Theorem 39. The proof relies on the technical results of Section 3. As an application, we prove, in Section 7 that a V-manifold structure is local. In Section 8, we similarly prove that a diffeological orbifold is the same thing as an orbifold structure in the sense of Haefliger $[\mathrm{H}]$.

The modern literature contains "higher" approaches to orbifolds, for example, through stacks or Lie groupoids. See $[\mathrm{DM}, \mathrm{Mo}, \overline{\mathrm{Me}}, \overline{\mathrm{BX}}]$. In these approaches, the finite group actions can be non-effective; the resulting structure is global, not local. A concrete relationship between the Lie groupoid approach to orbifolds and the diffeology approach to orbifolds is analyzed in a forthcoming paper by Y. Karshon and M. Zoghi.

We expect our diffeological approach to orbifolds to suffice for applications that do not involve global aspects of non-effective orbifolds. It is extremely easy to work with diffeology. Thus, when applicable, diffeology removes the language barrier to passing from manifolds to orbifolds.

\section{Diffeological orbifolds}

The notions of diffeology and diffeological spaces are due to Chen Che (under the name "differentiable spaces") and to Souriau [Sou1, Sou2. For details, see Igl1, Igl2,

A parametrization on a set $X$ is a map $p: U \rightarrow X$, where $U$ is an open subset of $\mathbb{R}^{n}$ for some $n$.

Definition 1 (Diffeology and diffeological spaces). A diffeology on a set $X$ is a set of parametrizations on $X$, whose elements are called plots, such that the following axioms hold:

Covering axiom: The constant parametrizations are plots.

Locality axiom: Let $p: U \rightarrow X$ be a parametrization. If every point in $U$ is contained in an open subset $V \subset U$ such that $\left.p\right|_{V}: V \rightarrow X$ is a plot, then $p$ is a plot.

Smooth compatibility axiom: Let $p: U \rightarrow X$ be a plot, $V$ an open subset of $\mathbb{R}^{m}$ for some $m$, and $F: V \rightarrow U$ a $C^{\infty}$ smooth map. Then $p \circ F$ is a plot.

A diffeological space is a set $X$ equipped with a diffeology.

Definition 2 (Differentiable maps). A map $f: X \rightarrow Y$ between diffeological spaces is called differentiable if the composition of $f$ with any plot of $X$ is a plot of $Y$. A map $f: X \rightarrow Y$ between diffeological spaces is called a diffeomorphism if $f$ is a bijection and both $f$ and $f^{-1}$ are differentiable.

Diffeological spaces with differentiable maps form a category.

Like the notion of a topology, the notion of a diffeology is very general. For instance, the set of all maps $U \rightarrow X$ satisfies the three diffeology axioms; it defines 
the coarse diffeology on $X$. The set of all locally constant maps $U \rightarrow X$ defines the discrete diffeology on $X$. Other diffeologies are described below. In each of the following definitions, we leave it to the reader to verify that the three diffeology axioms hold.

Example (Smooth manifolds). On a manifold $M$, the set of all $C^{\infty}$ smooth maps $p: U \rightarrow M$ is a diffeology. A map between manifolds is differentiable as a map of diffeological spaces if and only if it is smooth in the usual sense.

Remark 3. Any plot $U \rightarrow X$ of a diffeological space $X$ is differentiable, where the open subset $U \subset \mathbb{R}^{n}$ is taken with its manifold diffeology. This is the content of the smooth compatibility axiom.

Definition 4 (Quotient diffeology). Let $X$ be a diffeological space, let $\sim$ be an equivalence relation on $X$, and let $\pi: X \rightarrow Y:=X / \sim$ be the quotient map. The quotient diffeology on $Y$ is the diffeology in which $p: U \rightarrow Y$ is a plot if and only if each point in $U$ has a neighborhood $V \subset U$ and a plot $\tilde{p}: V \rightarrow X$ such that $\left.p\right|_{V}=\pi \circ \tilde{p}$.

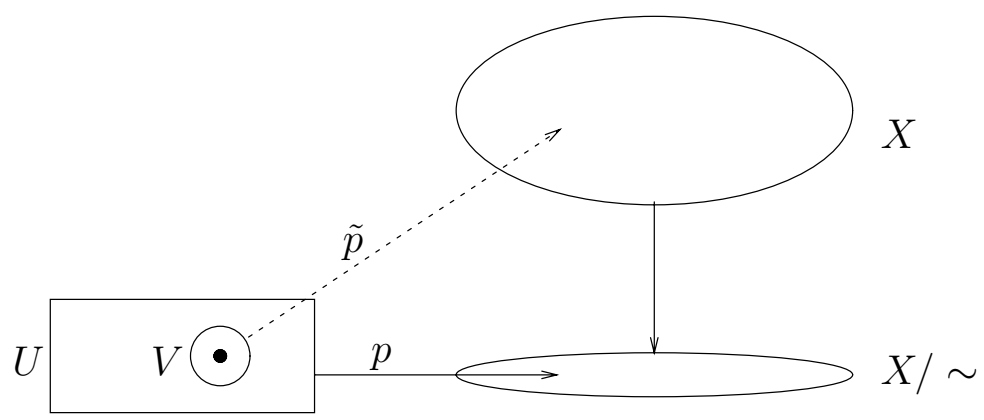

Figure 1. Quotient diffeology

Example. Let a finite group $\Gamma$ act on $\mathbb{R}^{n}$ by linear transformations. Then the quotient $\mathbb{R}^{n} / \Gamma$ is naturally a diffeological space.

Definition 5 (Local diffeomorphisms). A diffeological space $X$ is locally diffeomorphic to a diffeological space $Y$ at a point $x \in X$ if and only if there exists a subset $A$ of $X$, containing $x$, and there exists a one-to-one function $f: A \rightarrow Y$ such that

(1) for any plot $p: U \rightarrow X$, the composition $f \circ p$ is a plot of $Y$;

(2) for any plot $q: V \rightarrow Y$, the composition $f^{-1} \circ q$ is a plot of $X$.

Remark. The condition that the maps $f \circ p$ and $f^{-1} \circ q$ be plots includes the requirement that the domains of these maps, $p^{-1}(A)$ and $q^{-1}(f(A))$, be open.

Also see Remark 9 ,

Example. An $n$-dimensional manifold can be redefined as a diffeological space which is locally diffeomorphic to $\mathbb{R}^{n}$ at each point. Notice that being a manifold is a property of the preexisting diffeology; there is no need for any additional structure. 
Taking a similar approach to the notion of orbifolds, we make the following definition:

Definition 6 (Diffeological orbifolds). A diffeological orbifold is a diffeological space which is locally diffeomorphic at each point to a quotient $\mathbb{R}^{n} / \Gamma$, for some $n$, where $\Gamma$ is a finite group acting linearly on $\mathbb{R}^{n}$.

Remark. Diffeological orbifolds, with differentiable maps, form a subcategory of the category of diffeological spaces.

Definition 7 (D-topology). The D-topology on a diffeological space $X$ is the topology in which a subset $W \subset X$ is open if and only if $p^{-1} W$ is open for every plot $p: U \rightarrow X$. This is the finest topology on $X$ for which all plots are continuous. A differentiable map between diffeological spaces is always continuous with respect to their D-topologies. That is, we have a forgetful functor from diffeological spaces to topological spaces.

Example. On a smooth manifold, considered as a diffeological space, the Dtopology coincides with the standard topology. On $\mathbb{R}^{n} / \Gamma$, where $\Gamma \subset \mathrm{GL}_{n}(\mathbb{R})$ is a finite subgroup, the D-topology coincides with the standard quotient topology.

Definition 8 (Subset diffeology and diffeological subspaces). Let $X$ be a diffeological space, $A \subset X$ a subset, and $i: A \rightarrow X$ the inclusion map. Declare $p: U \rightarrow A$ to be a plot if and only if $i \circ p$ is a plot of $X$. This defines the subset diffeology on $A$. With this diffeology, $A$ is called a diffeological subspace of $X$.

In particular, any D-open subset of a diffeological space is naturally a diffeological space.

Remark 9. A diffeological space $X$ is locally diffeomorphic to a diffeological space $Y$ at a point $x \in X$ if and only if there exist a D-open subset $A$ of $X$ containing $x$ and a D-open subset $B$ of $Y$, equipped with their subset diffeologies, and a diffeomorphism $f: A \rightarrow B$.

If $\Gamma \subset \mathrm{GL}_{n}(\mathbb{R})$ is a finite subgroup, the open subsets of $\mathbb{R}^{n} / \Gamma$ are exactly the quotients $U / \Gamma$, where $U \subset \mathbb{R}^{n}$ is a $\Gamma$-invariant open set. The diffeology on $U / \Gamma$ can be considered either as the quotient diffeology of $U$ or, equivalently, as the subset diffeology of $\mathbb{R}^{n} / \Gamma$. Hence,

Remark 10. Any D-open subset of a diffeological orbifold is also a diffeological orbifold.

Example 11. Let a finite group $G$ act on a manifold $M$. Then the quotient $M / G$ is a diffeological orbifold. More generally, if a Lie group $G$ acts on a manifold $M$ properly and with finite stabilizers, then the quotient $M / G$ is a diffeological orbifold.

Proof. This is an immediate consequence of the slice theorem, by which a neighborhood of each $G$-orbit is equivariantly diffeomorphic to $G \times_{H} D$, where $H \subset G$ is the stabilizer of a point in the orbit, acting linearly on a disk $D$. See $\mathrm{Kz}$ for compact (in particular, finite) group actions and $[\mathrm{P}$ for proper group actions.

Lemma 12. Let $X$ be a diffeological space and $\mathcal{U}$ an open cover of $X$ with respect to the D-topology. Then the diffeology of $X$ is uniquely determined by the diffeology of the sets $U \in \mathcal{U}$. 


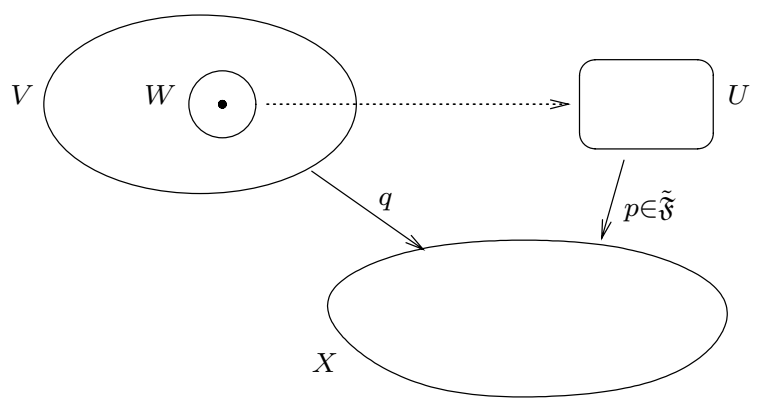

Figure 2. Generating family

Proof. This follows from Definition 7 and the locality axiom of a diffeology.

Definition 13. Let $\mathfrak{F}$ be a family of parametrizations on a set $X$. Let $\tilde{\mathfrak{F}}$ be the union of $\mathfrak{F}$ with the set of all constant parametrizations on $X$. Declare $q: V \rightarrow X$ to be a plot if and only if each point in $V$ has a neighborhood $W \subset V$, a parametrization $p: U \rightarrow X$ in $\tilde{\mathfrak{F}}$, and a smooth map $F: W \rightarrow U$, such that $\left.q\right|_{W}=p \circ F$. This defines the diffeology generated by $\mathfrak{F}$. See Figure 2 ,

We end this section with a characterization of diffeological orbifolds:

Lemma 14. A diffeological space $X$ is a diffeological orbifold if and only if for every $x \in X$ and open neighborhood $O$ of $x$ there exists a finite group $\Gamma$ acting on $\mathbb{R}^{n}$ linearly and a map $\varphi: \mathbb{R}^{n} \rightarrow X$ whose image is contained in $O$, such that $\varphi(0)=x$, and such that $\varphi$ induces a diffeomorphism of $\mathbb{R}^{n} / \Gamma$ with a neighborhood of $x$ in $X$.

Lemma 14 is a consequence of the slice theorem. See Definition 6 and the proof of Example 11. For completeness, let us also give a more elementary proof of this fact.

For any finite subgroup $\Gamma \subset \mathrm{GL}_{n}(\mathbb{R})$, let $\|\cdot\|_{\Gamma}$ be the norm on $\mathbb{R}^{n}$ that is induced from the inner product $\sum_{\gamma \in \Gamma}\langle\gamma u, \gamma v\rangle$ where $\langle\cdot, \cdot\rangle$ is the standard inner product. Then $\|\cdot\|_{\Gamma}$ is $\Gamma$-invariant.

Lemma 15. Let $\Gamma$ be a finite group, acting linearly on $\mathbb{R}^{n}$. Let $u \in \mathbb{R}^{n}$ be a point and let $\Gamma_{u}=\{\gamma \in \Gamma \mid \gamma \cdot u=u\} \subset \Gamma$ be its stabilizer. Let $U \subset \mathbb{R}^{n}$ be an open $\Gamma$-invariant subset containing $u$. Then there exists a $\Gamma_{u}$-equivariant open map

$$
\tilde{f}: \mathbb{R}^{n} \rightarrow U
$$

that sends the origin to $u$, is a diffeomorphism with its image, and such that the induced map

$$
\mathbb{R}^{n} / \Gamma_{u} \rightarrow U / \Gamma
$$

is a diffeomorphism with its image.

Proof. Let $\epsilon>0$ be sufficiently small so that

$$
B(u, \epsilon):=\left\{v \in \mathbb{R}^{n} \mid\|v-u\|_{\Gamma}<\epsilon\right\}
$$

is contained in $U$ and so that $2 \epsilon<\|\gamma \cdot u-u\|$ for all $\gamma \in \Gamma \backslash \Gamma_{u}$. Then the balls $B(u, \epsilon)$ and $\gamma \cdot B(u, \epsilon)$ are equal if $\gamma \in \Gamma_{u}$ and disjoint if $\gamma \in \Gamma \backslash \Gamma_{u}$. See Figure 3, 
where the $\gamma_{i}$ represent the non-trivial cosets in $\Gamma / \Gamma_{u}$. It follows that the inclusion $\operatorname{map} B(u, \epsilon) \hookrightarrow \mathbb{R}^{n}$ induces a one-to-one map

$$
B(u, \epsilon) / \Gamma_{u} \rightarrow U / \Gamma
$$

The vertical arrows in the diagram

$$
\begin{array}{cccc}
B(u, \epsilon) & \hookrightarrow & U \\
\downarrow & & \downarrow \\
B(u, \epsilon) / \Gamma_{u} & \rightarrow & U / \Gamma
\end{array}
$$

generate the quotient diffeologies; it follows that the map (1) is a diffeomorphism with its image. Let

$$
s: \mathbb{R}^{n} \rightarrow B(0, \epsilon)
$$

be a $\Gamma_{u}$-equivariant diffeomorphism. The map

$$
\tilde{f}(x)=u+s(x)
$$

has the desired properties.
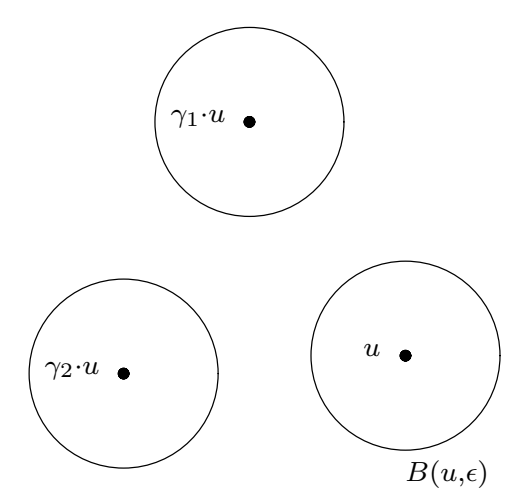

FiguRE 3. Neighborhood of a $\Gamma$-orbit

Proof of Lemma 14. The lemma follows immediately from Definition 6 and Lemma 15.

\section{LIFTING DIFFEOMORPHISMS OF FINITE QUOTIENTS}

In this section we show that a diffeomorphism (in the diffeological sense) of quotient spaces whose domain is $\mathbb{R}^{n} / \Gamma$, where $\Gamma \subset \mathrm{GL}_{n}(\mathbb{R})$ is a finite subgroup, always lifts to an equivariant diffeomorphism.

We note that this is not always true for differentiable maps that are not diffeomorphisms:

Example 16. Let $\mathbb{Z} / 2 \mathbb{Z}$ act on $\mathbb{R}^{2}$ by $(x, y) \mapsto \pm(x, y)$. Consider a map from $\mathbb{R}^{2}$ to $\mathbb{R}^{2} /(\mathbb{Z} / 2 \mathbb{Z})$ that is given by

$$
(r \cos \theta, r \sin \theta) \mapsto[g(r) \cos (\theta / 2), g(r) \sin (\theta / 2)],
$$

where $g$ is a function that vanishes near $r=0$ but does not vanish everywhere. This map is well defined, is differentiable, but does not lift to a differentiable (or even continuous) map from $\mathbb{R}^{2}$ to $\mathbb{R}^{2}$. 
Also see Examples 24 and 25.

The following result is a crucial ingredient in our analysis:

Lemma 17. Let $\Gamma \subset \mathrm{GL}_{n}(\mathbb{R})$ be a finite subgroup. Let $U \subset \mathbb{R}^{n}$ be a connected open subset. Let

$$
h: U \rightarrow \mathbb{R}^{n}
$$

be a continuously differentiable map that sends each point to a point in the same $\Gamma$-orbit. Then there exists a unique element $\gamma \in \Gamma$ such that

$$
h(x)=\gamma x
$$

for all $x \in U$.

Proof. For each $x \in U$ there exists $\gamma \in \Gamma$ such that (2) holds. We need to show that it is possible to choose the same $\gamma$ for all $x$.

Let $U_{0}$ denote the set of points in $U$ whose $\Gamma$-stabilizer is trivial. For $x \in U_{0}$, the element $\gamma \in \Gamma$ that satisfies (2) is unique. Because $h$ is continuous, this element is the same for all the points in the same connected component of $U_{0}$. For each connected component $A$ of $U_{0}$, denote by $\gamma_{A}$ this element of $\Gamma$, so that

$$
h(x)=\gamma_{A} x \quad \text { for all } x \in A .
$$

Then, because this is a linear map,

$$
d_{x} h=\gamma_{A} \quad \text { for all } x \in A,
$$

and because $h$ is continuously differentiable,

$$
d_{x} h=\gamma_{A} \quad \text { for all } \quad x \in \operatorname{closure}(A) .
$$

Because $U_{0}$ is obtained from $U$ by removing a finite union of proper subspaces, namely, the fixed point sets of the elements $\gamma \in \Gamma$ other than the identity, $U_{0}$ is dense in $U$. Because $U$ is connected, it follows that all the $\gamma_{A}$ 's are equal to each other.

Remark 18. In the above lemma, if $\Gamma$ contains no reflections, it is enough to assume that $h$ is continuous: if $\Gamma$ contains no reflections, the set $U_{0}$ is connected, so there exists $\gamma$ such that (2) holds for all $x \in U_{0}$. Because $U_{0}$ is dense and $h$ is continuous, (2) holds for all $x \in U$.

We also need the following topological fact:

Lemma 19. Let $\Gamma \subset \mathrm{GL}_{n}(\mathbb{R})$ be a finite subgroup. Let $U \subset \mathbb{R}^{n}$ be a $\Gamma$-invariant subset that contains the origin. If $U / \Gamma$ is connected, then $U$ is connected.

Proof. Suppose that $W \subset U$ is both open and closed in $U$. After possibly switching between $W$ and $U \backslash W$ we may assume that 0 is in $W$. Then each of the sets $\gamma \cdot W$, for $\gamma \in \Gamma$, contains 0 and is both open and closed in $U$. The intersection

$$
\bigcap_{\gamma \in \Gamma} \gamma \cdot W
$$

is $\Gamma$-invariant, and its image in $U / \Gamma$ is both open and closed and is non-empty. Because $U / \Gamma$ is connected, this image is all of $U / \Gamma$. Because the intersection (3) is $\Gamma$-invariant and its image is all of $U / \Gamma$, it follows that this intersection is equal to $U$. Thus, $W=U$.

The following three lemmas show that a diffeomorphism of finite quotients locally lifts to equivariant diffeomorphisms. 
Notation. We will denote the image of the origin under the quotient map $\mathbb{R}^{n} \rightarrow$ $\mathbb{R}^{n} / \Gamma$ by the symbol 0 and will still call it "the origin".

Lemma 20. Let $\Gamma \subset \mathrm{GL}_{n}(\mathbb{R})$ and $\Gamma^{\prime} \subset \mathrm{GL}_{n^{\prime}}(\mathbb{R})$ be finite subgroups. Let $U \subset \mathbb{R}^{n}$ and $U^{\prime} \subset \mathbb{R}^{n^{\prime}}$ be invariant open subsets that contain the origin. Let

$$
f: U / \Gamma \rightarrow U^{\prime} / \Gamma^{\prime}
$$

be a diffeomorphism of diffeological spaces such that $f(0)=0$. Let

$$
\tilde{f}: U \rightarrow U^{\prime}
$$

be a smooth map that lifts $f$. Then there exists a neighborhood of the origin on which $\tilde{f}$ is a diffeomorphism with an open subset of $U^{\prime}$. (In particular, it follows that $n=n^{\prime}$.)

Proof. By the definitions of the quotient diffeologies and of "diffeomorphism" (see Definitions 2 and (4), there exists an open $\Gamma^{\prime}$-invariant subset $V^{\prime} \subset U^{\prime}$, containing the origin, and a smooth map $\tilde{g}: V^{\prime} \rightarrow U$ that lifts the inverse map $f^{-1}: U^{\prime} / \Gamma^{\prime} \rightarrow$ $U / \Gamma$. We may choose $V^{\prime}$ to be connected, for instance, by taking it to be a ball around the origin with respect to a $\Gamma^{\prime}$-invariant metric. Let $V$ be the preimage of $f^{-1}\left(V^{\prime} / \Gamma^{\prime}\right)$ under the quotient map $U \rightarrow U / \Gamma$. Then $V / \Gamma$ is connected, because it is the image of the connected set $V^{\prime} / \Gamma^{\prime}$ under the continuous map $f^{-1}$. By Lemma 19. $V$ is also connected. We now have connected invariant open neighborhoods $V$ and $V^{\prime}$ of the origins in $\mathbb{R}^{n}$ and $\mathbb{R}^{n^{\prime}}$ and smooth maps

$$
\tilde{f}: V \rightarrow V^{\prime} \quad \text { and } \quad \tilde{g}: V^{\prime} \rightarrow V
$$

such that $\tilde{f}$ lifts $f$ and $\tilde{g}$ lifts $f^{-1}$. Without loss of generality, we assume that $U=V$ and $U^{\prime}=V^{\prime}$. We will show that $\tilde{f}$ is then a diffeomorphism.

The composition $\tilde{g} \circ \tilde{f}: V \rightarrow V$ sends each $\Gamma$-orbit to itself. By Lemma 17, there exists an element $\gamma$ of $\Gamma$ such that $\tilde{g} \circ \tilde{f}(x)=\gamma x$ for all $x \in V$. After replacing $\tilde{f}$ by $\tilde{f} \circ \gamma^{-1}$, we may assume that

$$
\left.\tilde{g} \circ \tilde{f}\right|_{V} \text { is the identity map. }
$$

Similarly, there exists an element $\gamma^{\prime} \in \Gamma^{\prime}$ such that

$$
\tilde{f} \circ \tilde{g}(x)=\gamma^{\prime} x \quad \text { for all } x \in V^{\prime} .
$$

Because $\tilde{g} \circ \tilde{f}$ is the identity, we also have that

$$
\tilde{f} \circ \tilde{g}(x)=x \quad \text { for all } x \in V^{\prime} \text { in the image of } \tilde{f},
$$

so that

$$
\gamma^{\prime} x=x \quad \text { for all } x \in V^{\prime} \text { in the image of } \tilde{f} .
$$

Because the image of $f$ is all of $V^{\prime} / \Gamma^{\prime}$, the image of $\tilde{f}$ contains an element $x$ whose stabilizer is trivial. By this and ([6),$\gamma^{\prime}=1$. So $\tilde{f}$ and $\tilde{g}$ are inverses of each other. In particular, $\tilde{f}$ is a diffeomorphism.

Lemma 21. Let $\Gamma \subset \mathrm{GL}_{n}(\mathbb{R})$ and $\Gamma^{\prime} \subset \mathrm{GL}_{n^{\prime}}(\mathbb{R})$ be finite subgroups. Let $U \subset \mathbb{R}^{n}$ and $U^{\prime} \subset \mathbb{R}^{n^{\prime}}$ be invariant open subsets. Let

$$
f: U / \Gamma \rightarrow U^{\prime} / \Gamma^{\prime}
$$

be a diffeomorphism of diffeological spaces. Let

$$
\tilde{f}: U \rightarrow U^{\prime}
$$


be a smooth map that lifts $f$. Then each point of $U$ has a neighborhood on which $\tilde{f}$ is a diffeomorphism with an open subset of $U^{\prime}$.

Consequently, $n=n^{\prime}$, and for each $u \in U$ the linear map $L=d_{u} \tilde{f}$ is invertible. The conjugation map $\gamma \mapsto L \gamma L^{-1}$ carries the stabilizer subgroup $\Gamma_{u}$ of $u$ to the stabilizer subgroup $\Gamma_{u^{\prime}}^{\prime}$ of $u^{\prime}=\tilde{f}(u)$; in particular, the stabilizer subgroups $\Gamma_{u}$ and $\Gamma_{u^{\prime}}^{\prime}$ are conjugate in $\mathrm{GL}_{n}(\mathbb{R})$.

Proof. Let $u \in U$ and $u^{\prime}=\tilde{f}(u)$. By Lemma 15, there exists a $\Gamma_{u}$-equivariant map $\tilde{g}: \mathbb{R}^{n} \rightarrow U$ that sends the origin to $u$, is a diffeomorphism with an open subset of $U$, and such that the induced map $g: \mathbb{R}^{n} / \Gamma_{u} \rightarrow U / \Gamma$ is a diffeomorphism with an open subset of $U / \Gamma$. Similarly, there exists a $\Gamma_{u^{\prime}}^{\prime}$-equivariant map $\tilde{g}^{\prime}: \mathbb{R}^{n^{\prime}} \rightarrow U^{\prime}$ that sends the origin to $u^{\prime}$, is a diffeomorphism with an open subset of $U^{\prime}$, and such that the induced map $g^{\prime}: \mathbb{R}^{n^{\prime}} / \Gamma_{u^{\prime}}^{\prime} \rightarrow U^{\prime} / \Gamma^{\prime}$ is a diffeomorphism with an open subset of $U^{\prime} / \Gamma^{\prime}$. The composition $\left(\tilde{g}^{\prime}\right)^{-1} \circ \tilde{f} \circ \tilde{g}$ is a smooth map from a $\Gamma_{u}$-invariant open subset of $\mathbb{R}^{n}$ that contains the origin to a $\Gamma_{u^{\prime}}$-invariant open subset of $\mathbb{R}^{n^{\prime}}$ that contains the origin. It sends 0 to 0 , and it lifts the diffeomorphism $\left(g^{\prime}\right)^{-1} \circ f \circ g$. The first part of the lemma follows by applying Lemma 20 to this composition.

Lemma 22. Let $\Gamma \subset \mathrm{GL}_{n}(\mathbb{R})$ and $\Gamma^{\prime} \subset \mathrm{GL}_{n^{\prime}}(\mathbb{R})$ be finite subgroups. Let $U \subset \mathbb{R}^{n}$ be an invariant connected open subset that contains the origin. Let

$$
f: U / \Gamma \rightarrow \mathbb{R}^{n^{\prime}} / \Gamma^{\prime}
$$

be a diffeomorphism of $U / \Gamma$ with an open subset of $\mathbb{R}^{n^{\prime}} / \Gamma^{\prime}$. Let

$$
\tilde{f}: U \rightarrow \mathbb{R}^{n^{\prime}}
$$

be a smooth map that lifts $f$. Then

(1) $\tilde{f}$ is one-to-one.

(2) Let $U^{\prime \prime}$ denote the preimage of $f(U / \Gamma)$ under the projection map $\mathbb{R}^{n^{\prime}} \rightarrow$ $\mathbb{R}^{n^{\prime}} / \Gamma^{\prime}$; note that $U^{\prime \prime} \subset \mathbb{R}^{n^{\prime}}$ is open. Then $\tilde{f}$ is a diffeomorphism of $U$ with a connected component of $U^{\prime \prime}$.

(3) Let $L=d_{0} \tilde{f}$. Note that, by Lemma 21, $h(\gamma)=L \gamma L^{-1}$ defines an isomorphism from $\Gamma$ to a subgroup of $\Gamma^{\prime}$. Then $\tilde{f}$ is h-equivariant.

Proof of (1). Because $\tilde{f}$ lifts $f$ and $f$ is one-to-one, if $\tilde{f}(u)=\tilde{f}(v)$, then there exists $\gamma \in \Gamma$ such that $v=\gamma \cdot u$. We need to show, for each $\gamma \in \Gamma$, that $\tilde{f}(u)=\tilde{f}(\gamma \cdot u)$ implies $\gamma \cdot u=u$.

Consider the set

$$
U_{\gamma}:=\{u \in U \mid \tilde{f}(\gamma \cdot u)=\tilde{f}(u)\} .
$$

Clearly, $U_{\gamma}$ is closed in $U$ and contains the fixed point set

$$
U^{\gamma}=\{u \in U \mid \gamma \cdot u=u\}
$$

We need to show that $U_{\gamma}=U^{\gamma}$.

Let $v$ be any point in $U_{\gamma}$, and let $\Gamma_{v}=\{g \in \Gamma \mid g \cdot v=v\}$ be its stabilizer.

- Suppose $\gamma \in \Gamma_{v}$. By Lemma 21, there exists a $\Gamma_{v}$-invariant neighborhood $V$ of $v$ on which $\tilde{f}$ is a diffeomorphism with an open subset of $\mathbb{R}^{n^{\prime}}$. In particular, $\left.\tilde{f}\right|_{V}$ is one-to-one, so

$$
U_{\gamma} \cap V=U^{\gamma} \cap V
$$


- Suppose $\gamma \in \Gamma \backslash \Gamma_{v}$. By Lemma 21, there exist neighborhoods $V_{1}$ of $v$ and $V_{2}$ of $\gamma \cdot v$ such that $\left.\tilde{f}\right|_{V_{1}}$ and $\left.\tilde{f}\right|_{V_{2}}$ are diffeomorphisms with open subsets of $\mathbb{R}^{n^{\prime}}$.

Let $V$ be a $\Gamma_{v}$-invariant connected neighborhood of $v$ contained in $V_{1} \cap$ $\tilde{f}^{-1}\left(\tilde{f}\left(V_{2}\right)\right)$. For instance, we may take $V$ to be a ball centered at $v$ with respect to an invariant metric. The map $u \mapsto\left(\left.\tilde{f}\right|_{V}\right)^{-1} \tilde{f}(\gamma \cdot u)$ from $V$ to $V$ sends each $\Gamma_{v}$-orbit to itself. Applying Lemma 17 to this map, we get an element $\gamma^{\prime} \in \Gamma_{v}$ such that

$$
\tilde{f}(\gamma \cdot u)=\tilde{f}\left(\gamma^{\prime} \cdot u\right)
$$

for all $u \in V$. By the previous paragraph applied to $\gamma^{\prime}$, for each $u \in V$, if $\tilde{f}\left(\gamma^{\prime} \cdot u\right)=\tilde{f}(u)$, then $\gamma^{\prime} \cdot u=u$. By this and (8),

$$
U_{\gamma} \cap V=U^{\gamma^{\prime}} \cap V \text {. }
$$

By (77) and (91), for every point $u \in U_{\gamma}$ there exists a neighborhood $V \subset U$ and a linear subspace $L \subseteq \mathbb{R}^{n}$ such that

$$
U_{\gamma} \cap V=L \cap V .
$$

If $u \notin$ interior $\left(U_{\gamma}\right)$, then $L$ is a proper subspace of $\mathbb{R}^{n}$, and, by (10), $V \cap$ interior $\left(U_{\gamma}\right)$ $=\varnothing$. Hence, interior $\left(U_{\gamma}\right)$ and $U \backslash \operatorname{interior}\left(U_{\gamma}\right)$ are both open in $U$. Because $U$ is connected, either $U_{\gamma}=U$ or $U_{\gamma}$ has an empty interior.

On a sufficiently small $\Gamma$-invariant neighborhood $V$ of the origin, $\tilde{f}$ is one-to-one (by Lemma 21), and so $U_{\gamma} \cap V=U^{\gamma} \cap V$ for all $\gamma \in \Gamma$. Because $\Gamma$ acts effectively, if $\gamma$ is not the identity element, then $U^{\gamma} \subset \mathbb{R}^{n}$ is a proper subspace. Combining this with the previous paragraph, we deduce that if $U_{\gamma}$ contains a non-empty open set, then $\gamma$ is the identity.

Let $v$ be any point in $U_{\gamma}$. Let $V$ be a $\Gamma_{v}$-invariant neighborhood of $v$ on which $\tilde{f}$ is one-to-one. As before, there exists $\gamma^{\prime} \in \Gamma_{v}$ such that $\tilde{f}\left(\gamma\left(\gamma^{\prime}\right)^{-1} u\right)=\tilde{f}(u)$ for all $u \in V$. (If $\gamma \in \Gamma_{v}$, then we may take $\gamma^{\prime}=\gamma$.) So the set $U_{\gamma\left(\gamma^{\prime}\right)^{-1}}$ contains the open set $V$. By the previous paragraph, $\gamma\left(\gamma^{\prime}\right)^{-1}=1$. So $\gamma \in \Gamma_{v}$. But for $\gamma \in \Gamma_{v}$ we have $V \cap U_{\gamma}=V \cap U^{\gamma}$.

We deduce that $U_{\gamma}=U^{\gamma}$.

A map is called proper if the preimage of any compact set is compact. A proper map to a first-countable Hausdorff space is a closed map.

Proof of (2). By Lemma 21, each point of $U$ has a neighborhood on which $\tilde{f}$ is a diffeomorphism with an open subset of $\mathbb{R}^{n^{\prime}}$. By this and part (1), $\tilde{f}$ is a diffeomorphism of $U$ with an open subset of $\mathbb{R}^{n^{\prime}}$.

Because $f(U / \Gamma)=U^{\prime \prime} / \Gamma^{\prime}$ and $\tilde{f}$ lifts $f$, we have $\tilde{f}(U) \subset U^{\prime \prime}$. Because $U$ is connected, $\tilde{f}(U)$ is contained in a connected component of $U^{\prime \prime}$; denote this component by $U^{\prime}$. Thus, $\tilde{f}$ is an open map from $U$ to $U^{\prime}$. We need to show that its image is all of $U^{\prime}$.

Consider the diagram

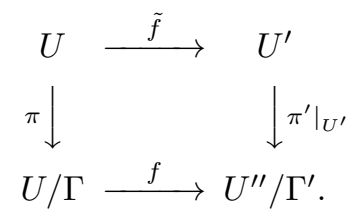


The projection maps $\pi: U \rightarrow U / \Gamma$ and $\pi^{\prime}: U^{\prime \prime} \rightarrow U^{\prime \prime} / \Gamma^{\prime}$ are proper. Because $U^{\prime} \subset U^{\prime \prime}$ is closed, $\left.\pi^{\prime}\right|_{U^{\prime}}: U^{\prime} \rightarrow U^{\prime \prime} / \Gamma$ is also proper. Because the maps $\pi,\left.\pi^{\prime}\right|_{U^{\prime}}$, and $f$ are proper, the map $\tilde{f}$ is also proper. Because $\tilde{f}: U \rightarrow U^{\prime}$ is open and proper and $U^{\prime}$ is connected, the image of $\tilde{f}$ is all of $U^{\prime}$.

Proof of (3). By Part (2), image $\tilde{f}=U^{\prime}$ is a connected component of $U^{\prime \prime}$, and $\tilde{f}: U \rightarrow U^{\prime}$ is a diffeomorphism. By Lemma 17, for each $\gamma \in \Gamma$ there exists a unique $\gamma^{\prime} \in \Gamma^{\prime}$ such that

$$
\tilde{f}\left(\gamma \cdot \tilde{f}^{-1}(x)\right)=\gamma^{\prime} \cdot x
$$

for all $x \in U^{\prime}$. Uniqueness implies that $\gamma \mapsto \gamma^{\prime}$ defines a group homomorphism

$$
h: \Gamma \rightarrow \Gamma^{\prime} \text {. }
$$

Then (11) becomes

$$
\tilde{f}(\gamma \cdot u)=\gamma^{\prime} \cdot \tilde{f}(u) \quad \text { for all } u \in U \text { and } \gamma^{\prime}=h(\gamma),
$$

which means that $\tilde{f}$ is $h$-equivariant.

Taking the derivatives at the origin of the left and right hand sides of (11) gives $L \gamma=\gamma^{\prime} L$, so that the group homomorphism $h$ coincides with the map $\gamma \mapsto L \gamma L^{-1}$ of Lemma 21.

We now reach the main result of this section.

Lemma 23 (Existence of global lifting). Let $\Gamma \subset \mathrm{GL}_{n}(\mathbb{R})$ and $\Gamma^{\prime} \subset \mathrm{GL}_{n^{\prime}}(\mathbb{R})$ be finite subgroups, let $U^{\prime} \subset \mathbb{R}^{n^{\prime}}$ be a $\Gamma^{\prime}$-invariant open subset, and let

$$
f: \mathbb{R}^{n} / \Gamma \rightarrow U^{\prime} / \Gamma^{\prime}
$$

be a diffeomorphism. Then there exists a smooth map $\tilde{f}: \mathbb{R}^{n} \rightarrow U^{\prime}$ that lifts $f$. Moreover, if $\tilde{f}$ is a smooth map that lifts $f$, then there exists a group isomorphism $h: \Gamma \rightarrow \Gamma^{\prime}$ such that $\tilde{f}$ is an h-equivariant diffeomorphism of $\mathbb{R}^{n}$ with a connected component of $U^{\prime}$.

Proof. For each $u \in \mathbb{R}^{n}$ and $r>0$, let

$$
B(u, r)=\left\{v \in \mathbb{R}^{n} \mid\|v-u\|_{\Gamma}<r\right\}
$$

denote the ball around $u$ of radius $r$ with respect to the $\Gamma$-invariant norm $\|\cdot\|_{\Gamma}$. By the definition of the quotient diffeologies, there exist $\rho>0$ and a smooth map

$$
\tilde{f}_{\rho}: B(0, \rho) \rightarrow U^{\prime}
$$

that lifts $f$. Consider the set

$$
\begin{aligned}
& \mathcal{R}=\{r \mid \text { there exists a smooth map } \tilde{f}: B(0, r) \rightarrow U^{\prime} \\
&\text { that lifts } \left.f \text { and extends } \tilde{f}_{\rho}\right\} .
\end{aligned}
$$

The set $\mathcal{R}$ is non-empty, because it contains $\rho$. Clearly, if $r \in \mathcal{R}$ and $\rho \leq r^{\prime}<r$, then $r^{\prime} \in \mathcal{R}$. Let us show that $\mathcal{R}$ is an open subset of $[\rho, \infty)$.

Fix any $r \in \mathcal{R}$. Let

$$
\tilde{f}: B(0, r) \rightarrow U^{\prime}
$$

be a smooth map that lifts $f$ and extends $\tilde{f}_{\rho}$. By Lemma 22, $\tilde{f}$ is a diffeomorphism of $B(0, r)$ with an open subset of $U^{\prime}$.

Let

$$
S(0, r)=\partial B(0, r)=\left\{v \in \mathbb{R}^{n} \mid\|v\|_{\Gamma}=r\right\} .
$$


By the definition of the quotient diffeologies and by Lemma 21, for each $x \in S(0, r)$ there exist $\epsilon>0$ and a lifting $B(x, \epsilon) \rightarrow U^{\prime}$ of $f$ which is a diffeomorphism with an open subset of $U^{\prime}$. Because $S(0, r)$ is compact, it can be covered by a finite number of such balls, $B_{i}=B\left(x_{i}, \epsilon_{i}\right)$. For each $i=1, \ldots, m$, let

$$
\tilde{f}_{i}: B_{i} \rightarrow U^{\prime} \quad, \quad i=1, \ldots, m
$$

be a lifting of $f$ which is a diffeomorphism with an open subset of $U^{\prime}$. Fix any $i$. By Lemma 17, there exists an element $\gamma \in \Gamma^{\prime}$ such that $\tilde{f}_{i} \circ \tilde{f}^{-1}(x)=\gamma \cdot x$ for all $x \in \tilde{f}\left(B_{i} \cap B(0, r)\right)$.

Replacing $\tilde{f}_{i}$ by $\gamma^{-1} \circ \tilde{f}_{i}$, we may assume that

$$
\tilde{f}_{i}=\tilde{f} \text { on } B_{i} \cap B(0, r) .
$$

Suppose that $B_{i} \cap B_{j}$ is non-empty. By Lemma [17, there exists an element $\gamma \in \Gamma^{\prime}$ such that

$$
\tilde{f}_{i} \tilde{f}_{j}^{-1}(x)=\gamma \cdot x \quad \text { for all } x \in \tilde{f}_{j}\left(B_{i} \cap B_{j}\right) .
$$

Because $B_{i}$ and $B_{j}$ are centered on $S(0, r)$ and $B_{i} \cap B_{j}$ is non-empty, the triple intersection $B(0, r) \cap B_{i} \cap B_{j}$ is also non-empty. By (14) applied to $i$ and $j$,

$$
\tilde{f}_{i} \tilde{f}_{j}^{-1}(x)=x \quad \text { for all } x \in \tilde{f}_{j}\left(B_{i} \cap B_{j} \cap B(0, r)\right) .
$$

Because the set $\tilde{f}_{j}\left(B_{i} \cap B_{j} \cap B(0, r)\right)$ is open and non-empty, it contains an element $x$ whose $\Gamma^{\prime}$-stabilizer is trivial. For this $x$, (15) and (16) imply that $\gamma=1$. So

$$
\tilde{f} \cup \tilde{f}_{1} \cup \ldots \cup \tilde{f}_{m}: B(0, r) \cup B_{1} \cup \ldots \cup B_{m} \rightarrow \tilde{U}^{\prime}
$$

is a well-defined lifting of $f$ which extends $\tilde{f}_{\rho}$. Because $B(0, r) \cup B_{1} \cup \ldots \cup B_{m}$ contains a ball of radius greater than $r$, this shows that the set $\mathcal{R}$, defined in (13), is an open subset of $[\rho, \infty)$.

For each $r \in \mathcal{R}$, let

$$
\tilde{f}_{r}: B(0, r) \rightarrow \tilde{U}^{\prime}
$$

be a smooth map that lifts $f$ and extends $\tilde{f}_{\rho}$. By Lemma 17, for any $r_{1}<r_{2}$ in $\mathcal{R}$ there exists an element $\gamma \in \Gamma^{\prime}$ such that

$$
f_{r_{2}} \circ f_{r_{1}}^{-1}(x)=\gamma \cdot x \quad \text { for all } x \in \tilde{f}_{r_{1}}\left(B\left(0, r_{1}\right)\right) .
$$

Because $\tilde{f}_{r_{1}}$ and $\tilde{f}_{r_{2}}$ both extend $\tilde{f}_{\rho}$, we have

$$
f_{r_{2}} \circ f_{r_{1}}^{-1}(x)=x \quad \text { for all } x \in \tilde{f}_{r_{1}}(B(0, \rho)) .
$$

By Lemma 21, the map $\tilde{f}_{r_{1}}$ is open, so the set $\tilde{f}_{r_{1}}(B(0, \rho))$ contains an element $x$ whose $\Gamma^{\prime}$-stabilizer is trivial. For this $x$, (17) and (18) imply $\gamma=1$. So $\left.\tilde{f}_{r_{2}}\right|_{B\left(0, r_{1}\right)}=$ $\tilde{f}_{r_{1}}$. Then

$$
\tilde{f}:=\bigcup_{r \in \mathcal{R}} \tilde{f}_{r}: \bigcup_{r \in \mathcal{R}} B(0, r) \rightarrow \tilde{U}^{\prime}
$$

is a well-defined lifting of $f$ which extends $\tilde{f}_{\rho}$.

This implies that if the set $\mathcal{R}$ defined in (13) is bounded, then it has a maximum. But since $\mathcal{R}$ is open in $[\rho, \infty)$, it cannot have a maximum. Since $r \in \mathcal{R}$ implies that $r^{\prime} \in \mathcal{R}$ for all $0<r^{\prime}<r$, we deduce that $\mathcal{R}=[\rho, \infty)$. So the domain of (19) is all of $\mathbb{R}^{n}$, and

lifts $f$ and extends $\tilde{f}_{\rho}$.

$$
\tilde{f}: \mathbb{R}^{n} \rightarrow \tilde{U}^{\prime}
$$


The fact that $\tilde{f}$ is an equivariant diffeomorphism with a connected component of $U^{\prime}$ then follows from Lemma 22 .

According to the above results, a diffeomorphism between finite linear quotients of $\mathbb{R}^{n}$ lifts to an equivariant diffeomorphism (by Lemma 23), and this lifting is unique up to an action of the finite linear groups (see Lemma 17). The following examples show that this existence and uniqueness of equivariant liftings may fail for differentiable functions that are not diffeomorphisms.

In both of these examples, let $\rho_{n}: \mathbb{R} \rightarrow \mathbb{R}$ denote a smooth function that takes values between 0 and 1 , vanishes outside the interval $\left[\frac{1}{n+1}, \frac{1}{n}\right]$, and is not always zero.

Example 24. For any $\epsilon=\left(\epsilon_{1}, \epsilon_{2}, \ldots\right) \in\{1,-1\}^{\mathbb{N}}$, let

$$
f_{\epsilon}: \mathbb{R} \rightarrow \mathbb{R}
$$

be the function

$$
f_{\epsilon}(x)= \begin{cases}\epsilon_{n} e^{-\frac{1}{x}} \rho_{n}(x) & \text { if } \frac{1}{n+1}<x \leq \frac{1}{n}, n \in \mathbb{N} \\ 0 & \text { if } x>1 \text { or } x \leq 0\end{cases}
$$

Then the functions $f_{\epsilon}$ give infinitely many inequivalent liftings of one function from $\mathbb{R}$ to $\mathbb{R} /\{ \pm 1\}$. Moreover, this remains true in any neighborhood of 0 .

Example 25. Let $f: \mathbb{R}^{2} \rightarrow \mathbb{R}^{2}$ be the function

$$
f(x, y)= \begin{cases}0 & \text { if } r>1 \text { or } r=0, \\ e^{-r} \rho_{n}(r)(r, 0) & \text { if } \frac{1}{n+1}<r \leq \frac{1}{n} \text { and } n \text { is even, } \\ e^{-r} \rho_{n}(r)(x, y) & \text { if } \frac{1}{n+1}<r \leq \frac{1}{n} \text { and } n \text { is odd }\end{cases}
$$

where $r=\sqrt{x^{2}+y^{2}}$. Fix any positive integer $m \geq 2$. The function $f$ descends to a function $[f]$ from $\mathbb{R}^{2} / \mathbb{Z} / m \mathbb{Z}$ to $\mathbb{R}^{2} / \mathbb{Z} / m \mathbb{Z}$. The function $[f]$ is differentiable because it has a smooth lifting, namely, $f$. On any annulus $\frac{1}{n+1}<r<\frac{1}{n}$ the function $[f]$ lifts to a smooth function that is equivariant with respect to a group homomorphism $h: \mathbb{Z} / m / Z \rightarrow \mathbb{Z} / m \mathbb{Z}$. However, for even $n$ the group homomorphism $h$ must be trivial, and for odd $n$ the group homomorphism $h$ must be the identity homomorphism. Consequently, there does not exist a neighborhood of 0 on which $[f]$ has an equivariant lifting with respect to one group homomorphism.

\section{Structure Groups of DifFeological orbifoldS}

Diffeology carries rich information; in particular, we now show that the structure groups are a diffeological invariant.

Lemma 26. Let $\Gamma \subset \mathrm{GL}_{n}(\mathbb{R})$ and $\Gamma^{\prime} \subset \mathrm{GL}_{n^{\prime}}(\mathbb{R})$ be finite subgroups. Suppose that there exist open subsets $V \subset \mathbb{R}^{n} / \Gamma$ and $V^{\prime} \subset \mathbb{R}^{n^{\prime}} / \Gamma^{\prime}$ that contain the origins and a diffeomorphism $\varphi: V \rightarrow V^{\prime}$ such that $\varphi(0)=0$. Then $n=n^{\prime}$, and $\Gamma$ and $\Gamma^{\prime}$ are conjugate in $\mathrm{GL}_{n}(\mathbb{R})$.

Proof. Let $U_{1} \subset \mathbb{R}^{n}$ and $U_{1}^{\prime} \subset \mathbb{R}^{n^{\prime}}$ denote the preimages of $V$ and $V^{\prime}$.

By the definitions of the quotient diffeologies and of "diffeomorphism", there exists an open neighborhood $U_{2}$ of 0 in $U_{1}$ and a smooth map $\tilde{\varphi}: U_{2} \rightarrow U_{1}^{\prime}$ that lifts $\varphi$. We may assume that $U_{2}$ is $\Gamma$-invariant (e.g., by shrinking it to a small ball around the origin with respect to a $\Gamma$-invariant metric). 
Let $U_{2}^{\prime} \subset U_{1}^{\prime}$ denote the preimage of $\varphi\left(U_{2} / \Gamma\right) \subset U_{1}^{\prime} / \Gamma^{\prime}$. Then $\varphi$ restricts to a diffeomorphism $\psi: U_{2} / \Gamma \rightarrow U_{2}^{\prime} / \Gamma^{\prime}$ such that $\psi(0)=0$, and $\tilde{\varphi}$ restricts to a smooth map $\tilde{\psi}: U_{2} \rightarrow U_{2}^{\prime}$ that lifts $\psi$.

By Lemma 21, $n=n^{\prime}$ and $\Gamma$ and $\Gamma^{\prime}$ are conjugate in $\mathrm{GL}_{n}(\mathbb{R})$.

Definition 27. Let $X$ be a diffeological orbifold and $x \in X$ a point. The structure group of $X$ at $x$ is a finite subgroup $\Gamma \subset \mathrm{GL}_{n}(\mathbb{R})$ such that there exists a diffeomorphism from $\mathbb{R}^{n} / \Gamma$ onto a neighborhood of $x$ in $X$ that sends the origin to $x$. By Lemma 14, such a group exists; by Lemma 26, such a group is unique up to conjugation in $\mathrm{GL}_{n}(\mathbb{R})$.

A singular point of a diffeological orbifold $X$ is a point whose structure group is non-trivial; a regular point, or a smooth point, is a point whose structure group is trivial. Because the structure group is unique up to conjugation, these notions are well defined. The set of regular points is open and dense; at these points, $X$ is a manifold. At a singular point, $X$ looks like a quotient $\mathbb{R}^{n} / \Gamma$ where $\Gamma$ is a non-trivial finite subgroup of $\mathrm{GL}_{n}(\mathbb{R})$.

To accurately state the relation of diffeological orbifolds to Satake's V-manifolds, we need to consider orbifolds whose structure groups do not contain reflections. (Also see Remark 33,)

Definition 28. A reflection is a linear map $\mathbb{R}^{n} \rightarrow \mathbb{R}^{n}$ whose fixed point set has codimension one. A reflection free diffeological orbifold is a diffeological space which is locally diffeomorphic at each point to a quotient $\mathbb{R}^{n} / \Gamma$ for some $n$, where $\Gamma$ is a finite group acting on $\mathbb{R}^{n}$ linearly and without reflections.

Remark 29. Notice that if a subgroup of $\mathrm{GL}_{n}(\mathbb{R})$ contains no reflections, then any subgroup of it or any group conjugate to it also contains no reflections. Thus, a diffeological orbifold is reflection free if and only if its structure groups contain no reflections. In particular, if $X$ is a reflection free diffeological orbifold, $\Gamma \subset \mathrm{GL}_{n}(\mathbb{R})$ is a finite subgroup, and $\psi: \mathbb{R}^{n} \rightarrow X$ induces a diffeomorphism from $\mathbb{R}^{n} / \Gamma$ onto an open subset of $X$, then $\Gamma$ contains no reflections.

The following characterization will be useful to connect our notion of a diffeological orbifold with Satake's notion of a V-manifold:

Proposition 30. A diffeological space is a reflection free diffeological orbifold if and only if each point has a neighborhood which is diffeomorphic to a quotient $\tilde{U} / \Gamma$, where $\tilde{U} \subset \mathbb{R}^{n}$ is a connected open subset and $\Gamma$ is a finite group of diffeomorphisms of $\tilde{U}$ whose fixed point sets have codimension $\geq 2$.

Proof. This follows immediately from Definition [, Example 11, and Remark 29.

\section{V-MANIFOLDS}

The notion of a V-manifold was introduced by Ichiro Satake in his two papers: On a Generalization of the Notion of Manifold [S1] and The Gauss-Bonnet Theorem for V-Manifolds [S2. Satake's definitions in [S2] slightly differ from those in [S1; they do lead to equivalent notions of "V-manifold", but this fact is not obvious. Thus Satake himself began the tradition in the literature of attributing to Satake a definition which a priori differs from his. We will follow the definitions of Satake's second paper, $\underline{\mathrm{S} 2}$. 
The local structure of a V-manifold is given by a local uniformizing system (l.u.s.); this can be thought of as a "local chart". The following definition is taken from [S2, pp. 465-466].

Definition 31. Let $M$ be a Hausdorff space and $U \subset M$ an open subset. A local uniformizing system (l.u.s.) for $U$ is a triple $(\tilde{U}, G, \varphi)$, where $\tilde{U}$ is a connected open subset of $\mathbb{R}^{n}$ for some $n$, where $G$ is a finite group of diffeomorphisms of $\tilde{U}$ whose fixed point sets have codimension $\geq 2$, and where $\varphi: \tilde{U} \rightarrow U$ is a map which induces a homeomorphism between $\tilde{U} / G$ and $U$.

Local uniformizing systems are patched together by injections; these can be thought of as the "transition maps". The following definition is taken from S2, p. 466]:

Definition 32. An injection from an l.u.s. $(\tilde{U}, G, \varphi)$ to an l.u.s. $\left(\tilde{U}^{\prime}, G^{\prime}, \varphi^{\prime}\right)$ is a diffeomorphism $\lambda$ from $\tilde{U}$ onto an open subset of $\tilde{U}^{\prime}$ such that

$$
\varphi=\varphi^{\prime} \circ \lambda \text {. }
$$

Remark 33. Eventually we would like to show that diffeological orbifolds are "the same as" V-manifolds. However, this is not quite true. One minor difference is that Satake only works with spaces that are Hausdorff, whereas in our definition of a diffeological orbifold we allow the D-topology to be non-Hausdorff. A more serious difference is that in his definition of an l.u.s., Satake only works with finite groups that act without reflections, whereas we allow arbitrary finite group actions. It appears that Satake's main usage of the reflection-free assumption is in [S1, Lemma 1, p. 360] and [S2, Lemma 1, p. 466], which assert this:

Lemma. Let $\lambda, \mu: \tilde{U} \rightarrow \tilde{U}^{\prime}$ be two injections. Then there exists a unique $\sigma^{\prime} \in G^{\prime}$ such that $\mu=\sigma^{\prime} \circ \lambda$.

However, this lemma remains true even if the finite groups contain reflections. This more general version of the lemma appeared in [MP, Appendix]. It can also be proved in a way similar to our proof of Lemma 17.

A V-manifold structure is given by a defining family; this can be thought of as an "atlas". The following two definitions are taken from [S2, p. 467].

Definition 34. Let $M$ be a Hausdorff space. A defining family on $M$ is a family $\mathcal{F}$ of l.u.s.'s for open subsets of $M$, satisfying conditions (1) and (2) below. An open subset $U \subset M$ is said to be $\mathcal{F}$-uniformized if there exists an l.u.s. $(\tilde{U}, G, \varphi)$ in $\mathcal{F}$ such that $\varphi(\tilde{U})=U$.

(1) Every point in $M$ is contained in at least one $\mathcal{F}$-uniformized open set. If a point $p$ is contained in two $\mathcal{F}$-uniformized open sets $U_{1}$ and $U_{2}$, then there exists an $\mathcal{F}$-uniformized open set $U_{3}$ such that $p \in U_{3} \subset U_{1} \cap U_{2}$.

(2) If $(\tilde{U}, G, \varphi)$ and $\left(\tilde{U}^{\prime}, G, \varphi^{\prime}\right)$ are l.u.s.'s in $\mathcal{F}$ and $\varphi(\tilde{U}) \subset \varphi^{\prime}\left(\tilde{U}^{\prime}\right)$, then there exists an injection $\lambda: \tilde{U} \rightarrow \tilde{U}^{\prime}$.

See Figure 4

One might naively attempt to declare two defining families to be equivalent if their union is a defining family. However, this is not an equivalence relation:

Example 35. Consider the annulus

$$
M=\left\{(x, y) \in \mathbb{R}^{2} \mid 0<x^{2}+y^{2}<1\right\} .
$$




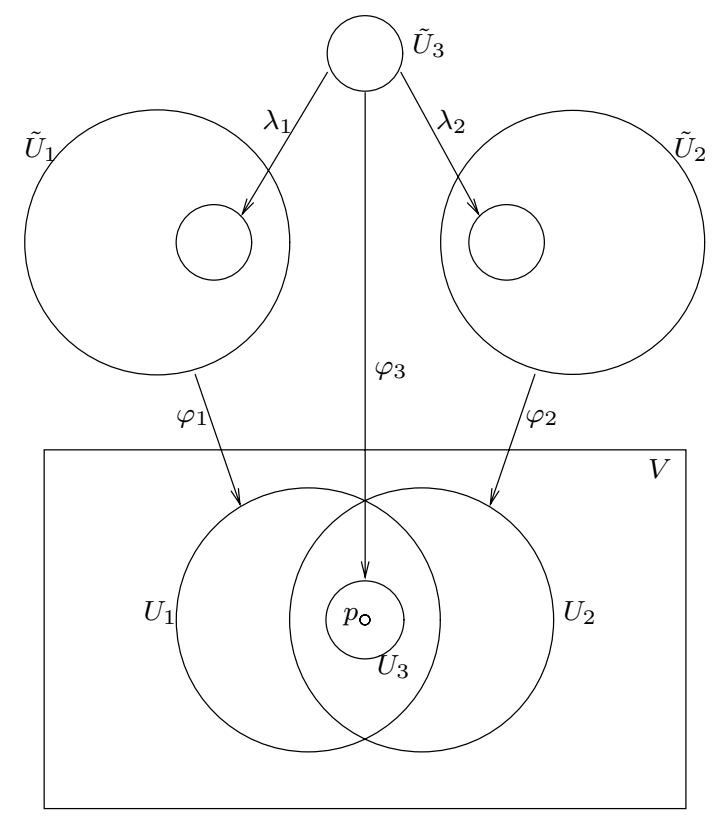

Figure 4. Defining family à la Satake

Consider the following two l.u.s.'s. The first consists of the annulus with the trivial group action and the identity map. The second consists of the annulus with the two-element group acting by $(x, y) \mapsto \pm(x, y)$ and with the map $\varphi(r \cos \theta, r \sin \theta)=$ $(r \cos 2 \theta, r \sin 2 \theta)$.

Let $\mathcal{F}$ consist of the first l.u.s., and let $\mathcal{F}^{\prime}$ consist of the second l.u.s. Let $\mathcal{F}^{\prime \prime}$ consist of all l.u.s.'s consisting of a disk that is contained in the annulus, with the trivial group action and the identity map. Then $\mathcal{F}, \mathcal{F}^{\prime}$, and $\mathcal{F}^{\prime \prime}$ are defining families on $M$. The unions $\mathcal{F} \cup \mathcal{F}^{\prime \prime}$ and $\mathcal{F}^{\prime} \cup \mathcal{F}^{\prime \prime}$ are defining families, but there is no defining family that contains both $\mathcal{F}$ and $\mathcal{F}^{\prime}$.

Definition 36. Two defining families, $\mathcal{F}$ and $\mathcal{F}^{\prime}$, are directly equivalent if there exists a third defining family that contains both $\mathcal{F}$ and $\mathcal{F}^{\prime}$. Two defining families $\mathcal{F}$ and $\mathcal{F}^{\prime}$ are equivalent if there exists a chain of direct equivalences starting with $\mathcal{F}$ and ending with $\mathcal{F}^{\prime}$.

The following definition is taken from [S2, p. 467, Definition 1 and footnote 1]:

Definition 37. A $V$-manifold is a Hausdorff space $M$ equipped with an equivalence class of defining families.

In what follows, whenever we choose a defining family on a V-manifold, we assume that this family belongs to the given equivalence class, unless otherwise stated.

\section{V-MANIFOLDS AS DIFFEOLOGICAL ORBIFOLDS}

In this section we describe the natural correspondence between Satake's Vmanifolds and diffeological orbifolds. 
Proposition 38. Let $M$ be a Hausdorff topological space. A defining family $\mathcal{F}$ on $M$ determines a diffeology, namely, the diffeology generated by the maps $\varphi: \tilde{U} \rightarrow$ $U$, for all $(\tilde{U}, G, \varphi) \in \mathcal{F}$.

(1) Let $\mathcal{F}$ be a defining family on $M$. Then, equipped with the diffeology generated by $\mathcal{F}, M$ is a reflection-free diffeological orbifold.

(2) Equivalent defining families $\mathcal{F}, \mathcal{F}^{\prime}$ on $M$ generate the same diffeology.

The natural diffeology on a V-manifold is the one determined by any defining family; see Definition 37 and Proposition 38.

Theorem 39. A V-manifold, with its natural diffeology, becomes a Hausdorff reflection-free diffeological orbifold. The diffeology uniquely determines the $V$-manifold structure. Every Hausdorff reflection-free diffeological orbifold arises in this way.

The rest of this section is devoted to the proof of Proposition 38 and Theorem 39 .

Lemma 40. Let $M$ be a Hausdorff topological space, let $U \subset U^{\prime} \subset M$ be open subsets, let $(\tilde{U}, G, \varphi)$ and $\left(\tilde{U}^{\prime}, G^{\prime}, \varphi^{\prime}\right)$ be l.u.s.'s for $U$ and $U^{\prime}$, and let $\lambda: \tilde{U} \rightarrow \tilde{U}^{\prime}$ be an injection. Then

(1) the image of $\lambda$ is a connected component of $\left(\varphi^{\prime}\right)^{-1}(U)$;

(2) $\lambda$ descends to a diffeomorphism from $\tilde{U} / G$ to an open subset of $\tilde{U}^{\prime} / G^{\prime}$.

Proof. From the definition of an l.u.s. and of an injection, it follows that $\lambda$ descends to a map $\bar{\lambda}: \tilde{U} / G \rightarrow \tilde{U}^{\prime} / G^{\prime}$ which is a homeomorphism with the open subset $\left(\varphi^{\prime}\right)^{-1}(U) / G^{\prime}$ of $\tilde{U}^{\prime} / G^{\prime}$.

Now consider the commuting diagram

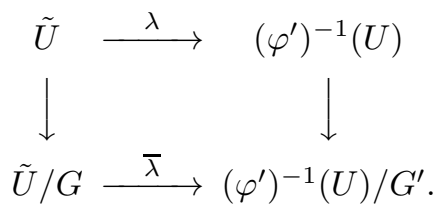

Because the quotient maps and the homeomorphism $\lambda$ are proper, $\lambda$ is proper, so its image is closed. But, by assumption, the image of $\lambda$ is also open and connected, so this image must be a connected component of $\left(\varphi^{\prime}\right)^{-1}(U)$.

Finally, consider the diagram

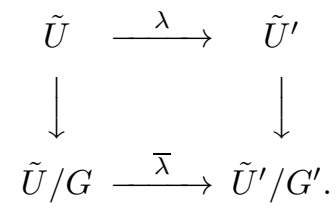

Because $\lambda$ is an open inclusion and the vertical arrows generate the quotient diffeologies, the map $\bar{\lambda}$ is a diffeomorphism with its image.

Corollary 41. Let $M$ be a Hausdorff space and $\mathcal{F}$ a defining family; equip $M$ with the diffeology generated by $\mathcal{F}$. Let $(\tilde{U}, G, \varphi)$ be an l.u.s. in $\mathcal{F}$. Then $U:=\varphi(\tilde{U}) \subset M$ is D-open, and the homeomorphism $\bar{\varphi}: \tilde{U} / G \rightarrow U$ induced by $\varphi$ is a diffeomorphism of diffeological spaces.

Part (1) of Proposition 38 follows from Proposition 30 and Corollary 41 Part (2) follows from Lemma 12, Lemma 40, and Corollary 41, 
Lemma 42. Let $M$ be a Hausdorff reflection-free diffeological orbifold. Let $\mathcal{U}$ be an open cover of $M$. Let $\mathcal{F}$ consist of all the triples $\left(\mathbb{R}^{n}, G, \psi\right)$, where $G \subset \mathrm{GL}_{n}(\mathbb{R})$ is a finite subgroup, $\psi: \mathbb{R}^{n} \rightarrow M$ induces a diffeomorphism from $\mathbb{R}^{n} / G$ onto an open subset of $M$, and there exists $U \in \mathcal{U}$ such that $\psi\left(\mathbb{R}^{n}\right) \subset U$. Then $\mathcal{F}$ is a defining family, and the set of $\mathcal{F}$-uniformized open sets is a basis to the topology of $M$.

Proof. By Remark 29, for each $\left(\mathbb{R}^{n}, G, \psi\right) \in \mathcal{F}$ the group $G$ is reflection free. Thus, $\left(\mathbb{R}^{n}, G, \psi\right)$ is an l.u.s. By Remark 10 and Lemma 14, the $\mathcal{F}$-uniformized open sets form a basis to the topology of $M$. So $\mathcal{F}$ satisfies Condition (1) of Definition 34 of a defining family. Condition (2) follows from Lemma 23. See Figure 5.

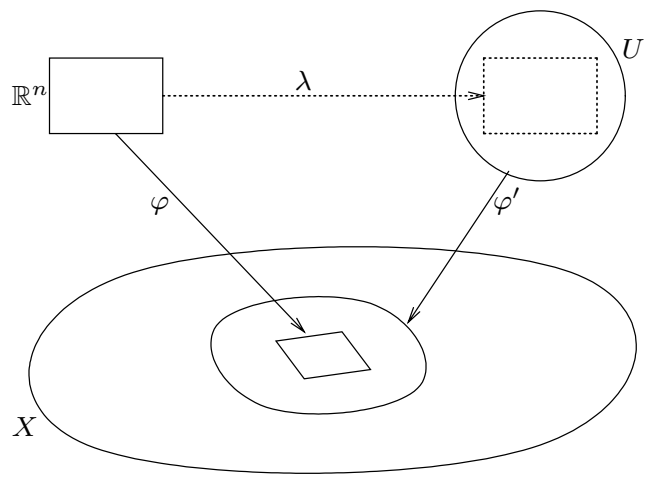

FIGURE 5

Proposition 43. Let $M$ be a Hausdorff topological space. If two defining families on $M$ generate the same diffeology, then they are equivalent.

Proof. Let $\mathcal{F}$ and $\mathcal{F}^{\prime}$ be defining families on $M$ that generate the same diffeology. Let $\mathcal{F}^{\prime \prime}$ be the defining family that is constructed as in Lemma 42 with respect to the open cover $\mathcal{U}=\left\{U \cap U^{\prime} \mid U\right.$ is $\mathcal{F}$-uniformized and $U^{\prime}$ is $\mathcal{F}^{\prime}$-uniformized $\}$. Let us show that $\mathcal{F} \cup \mathcal{F}^{\prime \prime}$ is a defining family.

Because the $\mathcal{F}^{\prime \prime}$-uniformized sets form a basis to the topology, $\mathcal{F} \cup \mathcal{F}^{\prime \prime}$ satisfies Condition (1) of Definition 34. To prove Condition (2), suppose that $\left(\tilde{U}_{i}, G_{i}, \varphi_{i}\right)$, for $i=1,2$, are l.u.s.'s in $\mathcal{F} \cup \mathcal{F}^{\prime \prime}$ with $\varphi\left(\tilde{U}_{1}\right) \subset \varphi\left(\tilde{U}_{2}\right)$. We will now show that there exists an injection from $\left(\tilde{U}_{1}, G_{1}, \varphi_{1}\right)$ to $\left(\tilde{U}_{2}, G_{2}, \varphi_{2}\right)$. If both l.u.s.'s are in $\mathcal{F}$, then the fact that $\mathcal{F}$ is a defining family implies that there exists such an injection. If $\left(\tilde{U}_{1}, G_{1}, \varphi_{1}\right) \in \mathcal{F}^{\prime \prime}$, then Lemma 23 implies that there exists such an injection. Suppose now that $\left(\tilde{U}_{1}, G_{1}, \varphi_{1}\right) \in \mathcal{F}$ and $\left(\tilde{U}_{2}, G_{2}, \varphi_{2}\right) \in \mathcal{F}^{\prime \prime}$.

By the definition of $\mathcal{F}^{\prime \prime}$ there exists an l.u.s. $\left(\tilde{U}_{3}, G_{3}, \varphi_{3}\right) \in \mathcal{F}$ such that $\varphi\left(\tilde{U}_{2}\right) \subset$ $\varphi\left(\tilde{U}_{3}\right)$. Because $\mathcal{F}$ is a defining family, there exists an open embedding $\alpha: \tilde{U}_{1} \rightarrow \tilde{U}_{3}$ such that $\varphi_{3} \circ \alpha=\varphi_{1}$. By Lemma 23, there exists a diffeomorphism $\beta$ from $\tilde{U}_{2}$ to a connected component of $\varphi_{3}^{-1}\left(\varphi_{2}\left(\tilde{U}_{2}\right)\right)$ such that $\varphi_{3} \circ \beta=\varphi_{2}$. By possibly composing with an element of $G_{3}$ we may assume that this connected component is the one that contains $\alpha\left(\tilde{U}_{1}\right)$. Then $\lambda:=\beta^{-1} \circ \alpha: \tilde{U}_{1} \rightarrow \tilde{U}_{2}$ is an injection, as required. See Figure 6 .

We have shown that $\mathcal{F} \cup \mathcal{F}^{\prime \prime}$ is a defining family. Applying a similar argument to $\mathcal{F}^{\prime}$ and $\mathcal{F}^{\prime \prime}$, we get a chain of direct equivalences: $\mathcal{F}, \mathcal{F}^{\prime \prime}, \mathcal{F}^{\prime}$. By Definition 36, the defining families $\mathcal{F}$ and $\mathcal{F}^{\prime}$ are equivalent. 


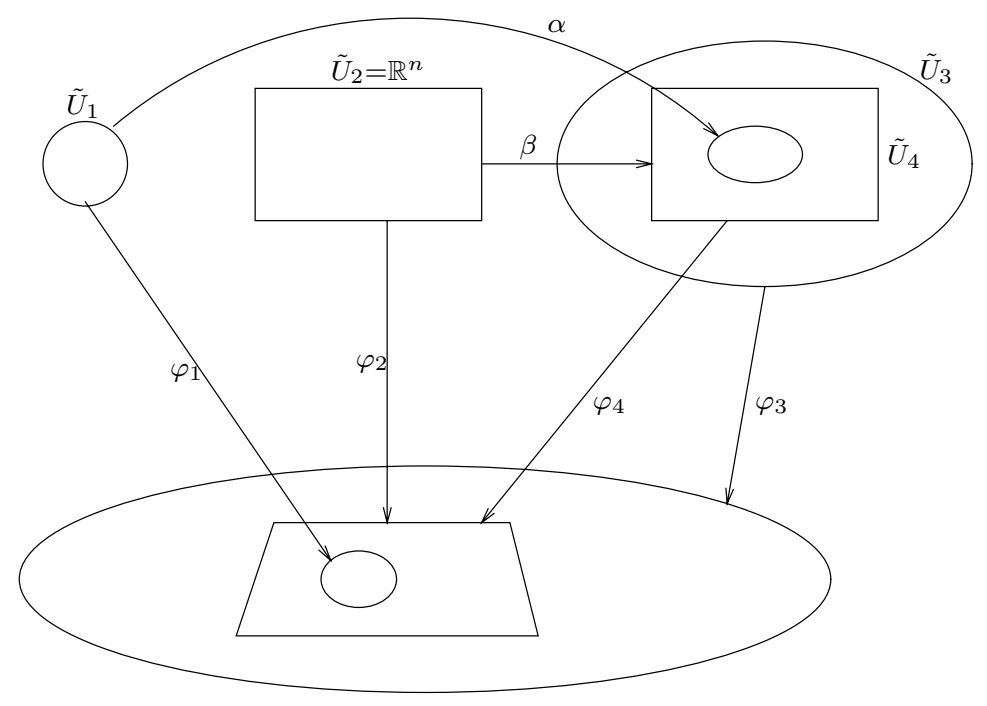

Figure 6

Proposition 44. Let $M$ be a Hausdorff reflection-free diffeological orbifold. Then there exists a defining family $\mathcal{F}$ on $M$ which generates its diffeology.

Proof. Let $\mathcal{F}$ consist of all the triples $\left(\mathbb{R}^{n}, G, \varphi\right)$, where $G \subset \mathrm{GL}_{n}(\mathbb{R})$ is a finite subgroup and where $\varphi: \mathbb{R}^{n} \rightarrow M$ induces a diffeomorphism from $\mathbb{R}^{n} / G$, equipped with its quotient diffeology, to an open subset of $M$, equipped with its subset diffeology. By Lemma 42 (with any $\mathcal{U}$ ), $\mathcal{F}$ is a defining family. By Corollary 41 and the definition of $\mathcal{F}$, the family $\mathcal{F}$ generates the diffeology on $M$.

Theorem 39 follows immediately from Propositions 43 and 44 .

\section{LOCALITY OF V-MANifOLD STRUCTURE}

Let $X$ be a topological space and $Y \subset X$ an open subset. Given a defining family $\mathcal{F}$ on $X$, its restriction $\left.\mathcal{F}\right|_{Y}$ to $Y$ is defined to be the set of all l.u.s.'s of the form $\left(\tilde{U}_{Y}, G,\left.\varphi\right|_{\tilde{U}_{Y}}\right)$, where $(\tilde{U}, G, \varphi)$ is an l.u.s. on $X$ and where $\tilde{U}_{Y}$ is a connected component of $\tilde{U} \cap \varphi^{-1}(Y)$. In this way, a V-manifold structure on $X$ naturally restricts to a $\mathrm{V}$-manifold structure on $Y$.

Given two defining families $\mathcal{F}_{1}$ and $\mathcal{F}_{2}$, if the restrictions $\left.\mathcal{F}_{1}\right|_{U}$ and $\left.\mathcal{F}_{2}\right|_{U}$ are equivalent for elements $U$ of an open covering $\mathcal{U}$ of $M$, a priori it is not clear that $\mathcal{F}_{1}$ and $\mathcal{F}_{2}$ themselves are equivalent. Also, given defining families $\mathcal{F}_{U}$ for $U \in \mathcal{U}$, if the restrictions $\left.\mathcal{F}_{U}\right|_{U \cap U^{\prime}}$ and $\left.\mathcal{F}_{U^{\prime}}\right|_{U \cap U^{\prime}}$ are equivalent for every $U, U^{\prime} \in \mathcal{U}$, a priori it is not clear how to patch together the families $\mathcal{F}_{U}$ into a defining family on $M$. However, these locality properties do follow, immediately, from the fact that a Vmanifold structure is determined by the diffeology that it generates, yielding the following result:

Proposition 45. Let $M$ be a Hausdorff topological space and $\mathcal{U}$ an open cover.

A $V$-manifold structure on $M$ is uniquely determined by its restrictions to the open sets $U$ in $\mathcal{U}$. 
Suppose that we are given for each $U \in \mathcal{U}$ a V-manifold structure on $U$. Suppose that, for each $U$ and $U^{\prime}$ in $\mathcal{U}$, the restrictions to $U \cap U^{\prime}$ of the $V$-manifold structures on $U$ and on $U^{\prime}$ coincide. Then there exists a unique $V$-manifold structure on $M$ whose restriction to each $U \in \mathcal{U}$ is the given $V$-manifold structure on $U$.

\section{Relation with Haefliger's Definition}

In section 4 of $[\underline{\mathrm{H}}]$, Haefliger gives this definition.

The structure of a $\mathrm{V}$-manifold on $Q$ is given by an open covering $U_{i}$, each $U_{i}$ being the quotient of a ball $\widetilde{U}_{i}$ in $\mathbb{R}^{q}$ by a finite group $G_{i}$ of diffeomorphisms. The projection $\pi_{i}: \widetilde{U}_{i} \rightarrow U_{i}=\widetilde{U}_{i} / G_{i}$ is called a uniformizing map. If $\widetilde{u}_{i}$ and $\widetilde{u}_{j}$ are two points in $\widetilde{U}_{i}$ and $\widetilde{U}_{j}$ such that $\pi_{i}\left(\widetilde{u}_{i}\right)=\pi_{j}\left(\widetilde{u}_{j}\right)$, one assumes that there exists a diffeomorphism $g_{j i}$ from a neighborhood of $\widetilde{u}_{i}$ onto a neighborhood of $\widetilde{u}_{j}$ such that $\pi_{j} \circ g_{j i}=\pi_{i}$ on a neighborhood of $\widetilde{u}_{i}$.

Thus, Haefliger works with triples $\left(\widetilde{U}_{i}, G_{i}, \pi_{i}\right)$, where $G_{i}$ is a finite group of diffeomorphisms of $\widetilde{U}_{i}$ and the map $\pi_{i}: \widetilde{U}_{i} \rightarrow Q$ induces a homeomorphism $\bar{\pi}_{i}: \widetilde{U}_{i} / G_{i} \rightarrow$ $U_{i}$ onto an open subset $U_{i}$ of $Q$. He gives a compatibility condition for such triples.

Theorem 46. Let $\left\{\left(\widetilde{U}_{i}, G_{i}, \pi_{i}\right)\right\}$ be a collection that satisfies Haefliger's compatibility condition such that the sets $U_{i}$ cover $Q$. Then $Q$, equipped with the diffeology generated by the maps $\pi_{i}$, is a diffeological orbifold. Two such collections generate the same diffeology on $Q$ if and only if their union satisfies Haefliger's compatibility condition. Every diffeological orbifold is obtained in this way.

Proof. Haefliger's compatibility condition implies that the transition functions $\bar{\pi}_{j}{ }^{-1} \circ \bar{\pi}_{i}$ are differentiable in the diffeological sense. By Lemma 20 and the slice theorem, if $\bar{\pi}_{j}{ }^{-1} \circ \bar{\pi}_{i}$ is a diffeomorphism in the diffeological sense, then $\left(\widetilde{U}_{i}, G_{i}, \pi_{i}\right)$ and $\left(\widetilde{U}_{j}, G_{j}, \pi_{j}\right)$ are compatible in Haefliger's sense. These two facts imply the theorem.

Thus, an orbifold structure in Haefliger's sense is the same thing as a diffeological orbifold; hence, in the reflection-free case, it is also the same thing as Satake's Vmanifold. A priori this is not obvious from the definitions in [H] and [S1, S2].

\section{Acknowledgement}

The first author is grateful to the Hebrew University of Jerusalem for its hospitality that has led to this collaboration. The second author is grateful to Sue Tolman for convincing her, years ago, that she doesn't understand orbifolds. In her 1993 thesis [T] Tolman defined a cohomology theory for orbifolds and showed how to compute it for moduli spaces of complex curves.

\section{REFERENCES}

[BX] K. Behrend and P. Xu, Differentiable Stacks and Gerbes, arXiv:math/0605694v2.

[Che] K. T. Chen, Iterated path integral, Bull. Amer. Math. Soc. 83 no. 5 (1977), 831-879. MR0454968 (56:13210)

[DM] P. Deligne and D. Mumford, The irreducibility of the space of curves of given genus, Inst. Hautes Études Sci. Publ. Math. No. 36 (1969), 75-109. MR0262240 (41:6850)

[H] A. Haefliger, Groupoïdes d'holonomie et classifiants, Astérisque 116 (1984), pp. 70-97. $\operatorname{MR} 755163$ (86c:57026a) 
[Igl1] P. Iglesias, Fibrés difféologiques et homotopie, Thèse de doctorat.

[Igl2] P. Iglesias-Zemmour, Diffeology (draft), downloadable from <http://math.huji.ac. il/ ${ }^{\sim}$ piz/>.

[Kz] J. L. Koszul, Sur certains groupes de transformations de Lie, Colloque International du Centre National de la Recherche Scientifique 52 (1953), 137-141. MR0059919 (15:600g)

[Me] D. Metzler, Topological and Smooth Stacks, arXiv:math/0306176v1.

[Mo] I. Moerdijk, Orbifolds as Groupoids: an Introduction, in: Orbifolds in mathematics and physics (Madison, WI, 2001), 205-222, Contemp. Math. 310, Amer. Math. Soc., Providence, 2002. MR.1950948 (2004c:22003)

[MP] I. Moerdijk and D. A. Pronk, Orbifolds, sheaves and groupoids, K-Theory 12, 3-21, 1997. MR 1466622 (98i:22004)

[P] Richard Palais, On the existence of slices for actions of non-compact Lie Groups, Annals of Math. (2) 73 (1961), 295-323. MR0126506 (23:A3802)

[S1] I. Satake, On a generalization of the notion of manifold, Proceedings of the National Academy of Sciences 42 (1956), 359-363. MR0079769 (18:144a)

[S2] Ichiro Satake, The Gauss-Bonnet theorem for V-manifolds, Journal of the Mathematical Society of Japan, Vol. 9., No. 4 (1957), 464-492. MR.0095520 (20:2022)

[Sou1] J. M. Souriau, Groupes différentiels, Differential geometrical methods in mathematical physics (Proc. Conf., Aix-en-Provence/Salamanca, 1979), pp. 91-128, Lect. Notes in Math. 836, Springer, Berlin-New York, 1980. MR607688 (84b:22038)

[Sou2] J. M. Souriau, Groupes différentiels et physique mathématique, South Rhone seminar on geometry, II (Lyon, 1983), pp. 73-119, Travaux en Cours, Hermann, Paris, 1984. MR753860 (86a:58001)

[T] Susan Tolman, Ph.D. thesis, Harvard University, 1993.

Laboratory of Analysis, Topology and Probability - CNRS, 13453 Marseille Cedex 13, France - and - Department of Mathematics, The Hebrew University of Jerusalem, 91904 JeRUSAlEm, ISRAEL

E-mail address: piz@math.huji.ac.il

Department of Mathematics, The University of Toronto, 40 St. George Street, Toronto, Ontario, CANada M5S 2E4

E-mail address: karshon@math.toronto.edu

E-mail address: moshez@divmod.com 